\title{
GDP forecast of accommodation and catering industry in X city based on multi variable grey model _Energy saving development of accommodation and catering industry
}

\author{
Qian $\mathrm{Gao}^{1, *}$, Yunyun Liu ${ }^{2}$, Junyi Yang ${ }^{3}$, Yu Hong ${ }^{4}$, Lei Wen ${ }^{5}$ \\ ${ }^{1}$ Qian Gao, State Grid Jiangsu Electric Power, Nanjing City, China \\ ${ }^{2}$ Yunyun Liu, State Grid Suqian Power Supply Company, Suqian City, China \\ ${ }^{3}$ Junyi Yang, State Grid Jiangsu Electric Power, Nanjing City, China \\ ${ }^{4}$ Yu Hong, State Grid Jiangsu Electric Power, Nanjing City, China \\ ${ }^{5}$ Lei Wen, Southeast University, Nanjing City, China
}

\begin{abstract}
As the GDP of lodging and catering industry is generally quarterly or annual, the data volume is very small, so it is difficult to accurately predict the GDP of lodging and catering industry in this city for a city. However, a city can accurately predict the GDP development of the lodging and catering industry through electricity consumption. And the local government can formulate relevant policies to promote the stable development of lodging and catering industry and economy, and find the relationship between electricity consumption and GDP development of this industry, so as to achieve the purpose of low energy consumption and fast economic growth.t. This paper first conducted desensitization and normalization processing on the data, and then used Matlab to forecast the GDP of the lodging and catering industry in X City respectively by using GM $(1, N)$ grey model, multiple linear regression model and BP neural network model. The experimental results show that the GM $(1, \mathrm{~N})$ grey model is more accurate and reliable than the other two methods in the case of less data. This paper solves the problem that the GDP of accommodation and catering industry in a single city is inaccurate.
\end{abstract}

\section{Introduction}

In recent years, electricity data has attracted more and more attention from economists because of its real-time and high efficiency. Using electricity data to study economic structure and growth trend has become one of the hot topics in modern times.

In 2007, Sheng-tung Chen and Hsiao-i Kuo et al. studied the relationship between electricity consumption and GDP in ten Asian developing countries and concluded that there was a two-way long-term causal relationship between electricity consumption and economic growth ${ }^{[1]}$. A.Ciarreta and A.Zarraga investigated the electricity and GDP data of 12 European countries from 1970 to 2007 in 2010 and found that there was a short-term negative causal relationship between electricity consumption and GDP ${ }^{[2]}$. Yoo S H and Kwak S Y study seven South American countries the causality between electricity consumption and economic growth between the found that the causal relationship between electricity consumption and economic growth vary from country to country, in some countries, the electricity consumption of one-way directly affect economic growth, in some countries, the mutual influence between electricity consumption and economic relationship ${ }^{[3]} ;$ In the study on the relationship between China's electricity consumption and economic growth, Pan Kai found that there is a long-term equilibrium relationship between China's electricity consumption and economic development ${ }^{[4]}$. Li Xinying found in 2012 that the Granger causality between Xinjiang's electricity consumption and Xinjiang's economy is a one-way relationship from GDP to installed capacity and electricity consumption ${ }^{[5]}$. Luo Guanglei, Hu Xiguo and Zhao Yang predicted the development of electricity consumption and GDP of a city through a simple GM $(1,1)$ forecasting model in $2014{ }^{[6]}$. Sun Yan and Luo Xiaoqing found in 2016 that the marginal effect of electricity consumption on national economy is not fixed ${ }^{[7]}$. In 2017, He Yongxiu et al. showed that there was a Granger relationship between the total electricity consumption and economic development in Shanxi province, and analyzed the deep relationship between the primary, secondary and tertiary industries and electricity consumption, and put forward constructive suggestions for the industrial structure adjustment of Shanxi Province $^{[8]}$. Gao Qian and Liu Yunyun et al. proposed that the dynamic model could improve the prediction accuracy of GDP of the tertiary industry based on electricity consumption ${ }^{[9]}$. Chang Haotian proposed in 2020 that the accuracy of prediction could be improved 
by integrating data such as temperature, humidity and new users of Weibo on the basis of using electricity consumption to predict GDP ${ }^{[10]}$.

The above research fully shows that there is a deep relationship between electricity consumption and economic development, and it can predict economic development and analyze the state of economic structure according to electricity consumption. But the research is based on the study of some countries or some provinces, so the data is rich, because GDP statistics generally in the form of year or quarter, in terms of a single city GDP data of each industry is not enough, can't use in the study of above commonly used neural network method based on the electricity consumption of a specific industry GDP was analyzed and forecast. In the past studies, some scholars also used the grey prediction model to forecast GDP or electricity consumption, but these scholars either only predicted the development trend of GDP or electricity consumption alone, or because the model was too simple, they only considered the influence of electricity consumption on GDP without considering the influence of other factors ${ }^{[7],[11],[12]}$.

The lodging and catering industry is one of the important components of the tertiary industry. The healthy development of the lodging and catering industry is conducive to the healthy development of the economy. The effective prediction of the GDP of the lodging and catering industry can help the government implement effective policies to promote the healthy development of the lodging and catering industry. In order to accurately predict the GDP of the lodging and catering industry in a certain city, the multivariable grey prediction model

( GM $(1, N)$ grey model) is adopted in this paper. Compared with common neural network models, GM (1, $\mathrm{N})$ grey model requires less data and can obtain accurate prediction results with only a small amount of data. Compared with GM $(1,1)$ grey model, GM $(1, \mathrm{~N})$ grey model can take other factors into account, which is more reasonable and in line with the actual situation. Compared with linear regression model, GM $(1, \mathrm{~N})$ grey model is more reliable. In this paper, $\operatorname{GM}(1, N)$ grey model, Back Propagation neural network (BP neural network) and multiple linear regression model are used to predict the GDP of lodging and catering industry in X city respectively. The results show that the prediction results of $\operatorname{GM}(1, N)$ grey model is the most accurate and reliable.

\section{Materials and Methods}

In order to accurately use electricity to predict the GDP of lodging and catering industry.GM $(1, \mathrm{~N})$ model, BP network, Multiple linear regression are used to compare the experiments.

\subsection{The relationship between GDP and electricity consumption of accommodation catering industry in $\mathrm{X}$ city}

The development of the accommodation catering industry is fundamentally related to the overall development of the national economy. Its GDP is one of the components of the national GDP, and also reflects the national consumption level. It is another barometer of the national economic development. To accurately predict the GDP of the accommodation catering industry, government departments can timely implement effective policies to promote the healthy development of the accommodation catering industry. This paper mainly forecasts the GDP of accommodation catering industry in $\mathrm{X}$ city, but the temperature has a huge impact on the electricity consumption of accommodation catering industry, so the number of abnormal temperature weather is taken as the second independent variable to correct the electricity consumption.

In this paper, the GDP, electricity consumption and abnormal weather temperature of lodging and catering industry in X city (the abnormal weather temperature is the air temperature that can be turned on by enterprises and institutions under national regulations, that is, the air temperature is higher than $30^{\circ} \mathrm{C}$ or lower than $8^{\circ} \mathrm{C}$ ) are all from the official platform of $\mathrm{X}$ city, and there is no missing value in the sample. The GDP of accommodation catering industry in $\mathrm{X}$ city is calculated by year, and the electricity consumption is recorded in the database in real time. However, this paper forecasts the trend of accommodation catering industry GDP by electricity consumption and temperature. In order to increase the accuracy of prediction, the electricity consumption in this paper is also calculated by year. Due to the large gap between GDP and electricity consumption, it needs to be normalized. The trend chart formed after the treatment is shown in Figure 1. From Figure 1, it can be seen that the development trend of electricity consumption and GDP of accommodation catering industry is generally identical and highly correlated.

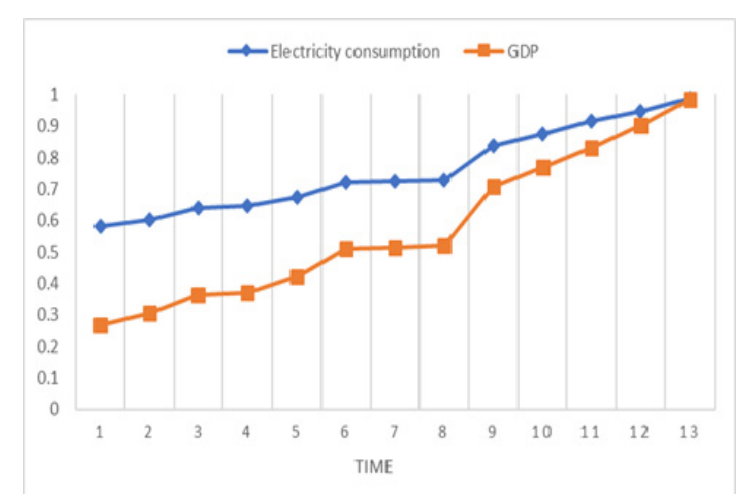

Fig. 1. Development trend of GDP and electricity consumption of accommodation and catering industry in $\mathrm{X}$ city 


\subsection{Methods describe}

\subsubsection{GM $(1, N)$ grey model}

Grey prediction model is to establish a grey differential prediction model through a small amount of incomplete information, to make a fuzzy long-term description of the law of development of things.

The basic steps of the multivariate grey prediction model are as follows:

1) Add up to generate new samples

2) Establish mathematical model

3) According to the model, $\hat{A}$ and $\hat{B}$ in the mathematical model are solved

4) The prediction formula is established to calculate the prediction error and comprehensive error when $\eta$ traverses from 1 to $M$

5) Select the $\eta$ value with the minimum comprehensive error, determine the best prediction formula, and finally get the corresponding results.

The mathematical model is as follows:

$$
\begin{aligned}
& \hat{X}^{(1)}(\eta)=e^{\lambda(k-\eta)} X^{(1)}(\eta)+\hat{A}^{-1}\left(e^{\lambda(k-\eta)}-I\right) \cdot \hat{B} \\
& k=1,2, \cdots m \quad \eta=1,2, \cdots m
\end{aligned}
$$

\subsubsection{The model of Multiple linear regression}

Multiple linear regression describes the dependence between a dependent variable and multiple independent variables by regression equations. The mathematical models of multiple and linear regressions are shown below:

$$
\mathrm{y}=\alpha_{0}+\alpha_{1} x_{1}+\cdots+\alpha_{n} x_{n}+\varepsilon
$$

In the formula, $y$ is the dependent variable, $x$ is the independent variable, $\alpha_{0}$ is the constant term, $\alpha_{1}, \cdots, \alpha_{n}$ is the partial regression coefficient , $\varepsilon$ is the random error and also known as the residual error, and $\varepsilon$ is the part of the change in $y$ that cannot be explained by the independent variable and obeys the $N\left(0, \sigma^{2}\right)$ distribution.

The multiple linear regression equation estimated by the sample is:

$$
\hat{y}=b_{0}+b_{1} x_{1}+\cdots+b_{n} x_{n}
$$

$\hat{y}$ is the average estimated value or average predicted value of the dependent variable $y$ when a set of fixed values of each $x$ is taken. $b_{0}, b_{1}, \cdots, b_{n}$ is the sample estimate of $\alpha_{0}, \alpha_{1}, \cdots, \alpha_{n}$.

Standardize the original data:

$$
x_{i}^{*}=\frac{x_{i}-\bar{x}_{i}}{S_{j}}
$$

Then the standardized data is used to fit the regression model, and the regression coefficient obtained at this time is denoted as $\mu_{0}, \mu_{1}, \cdots, \mu_{n}$, which is called standardized partial regression coefficient.

\subsubsection{The model of Back Propagation neural network}

Back Propagation neural network (BP neural network) was proposed by scientists led in 1986. It is a multi-layer feedforward neural network trained according to the error back propagation algorithm, and it is the most widely used neural network ${ }^{[13]}$.

The mathematical model of BP neural network is as follows:

$$
\begin{gathered}
\alpha(k)=W(k) \cdot s\left(V(k) \cdot z(k)+\mu_{1}\right)+\mu_{2} \\
z(k)=\left[v_{i}(k-1), \cdots, v_{i}(k-3), \mu_{i}(k-1), \cdots, \mu_{i}(k-3)\right]
\end{gathered}
$$

is the input of BP neural network. $W, V$ represent the weight matrix of BP neural network. $\mu_{1}, \mu_{2}$ represent thresholds. $s(\bullet)$ represent Sigmoid operator. $\alpha(k)$ is the estimate. The threshold and weight are constantly modified through back propagation to make the output optimal.

\section{Results and discussion}

In this paper, the GDP, electricity consumption, days with abnormal temperature and other data of the lodging and catering industry in X City from January 2006 to December 2018 were collected on annual basis. In order to eliminate the impact of currency devaluation, GDP was converted in 2006 as the base year. After removing outliers, normalization and desensitization, the data obtained were shown in Table 1, ES stands for electricity consumption, ATD is the number of days with abnormal temperatures:

Table 1. List of data.

\begin{tabular}{cccc}
\hline number & EC & GDP & ATD \\
\hline 1 & 0.584 & 0.268 & 135 \\
2 & 0.604 & 0.305 & 148 \\
3 & 0.640 & 0.364 & 126 \\
4 & 0.646 & 0.372 & 134 \\
5 & 0.673 & 0.423 & 131 \\
6 & 0.723 & 0.511 & 146 \\
7 & 0.726 & 0.516 & 164 \\
8 & 0.729 & 0.521 & 138 \\
9 & 0.838 & 0.708 & 130 \\
10 & 0.875 & 0.768 & 121 \\
11 & 0.916 & 0.832 & 135 \\
12 & 0.945 & 0.901 & 125 \\
13 & 0.989 & 0.983 & 165 \\
\hline
\end{tabular}




\subsection{Results}

\subsubsection{GM(1,N) grey model result}

Matlab was used for modeling with $\operatorname{GM}(1, N)$ grey model. The GDP of the lodging and catering industry in X City from 2006 to 2018 was taken as the dependent variable, and the electricity consumption and abnormal weather number were taken as independent variables to predict the trend of the lodging and catering industry GDP in X City. The data from 2006 to 2015 were used as the training set, and the data from 2016 to 2018 were used as the test set. The training results are shown in Table 2. It can be seen from Table 2 that the difference between the actual value and the simulated data are small, and the maximum relative error is $0.824 \%$, averaging relative simulation error is $0.34 \%$, simulation accuracy is relatively high, residuals are all below 0.005 , this means that the results are credible.

Table 2. Results of GM $(1, \mathrm{~N})$ grey model training.

\begin{tabular}{ccccc}
\hline \multicolumn{2}{c}{ number Actual GDP } & $\begin{array}{c}\text { Simulated } \\
\text { GDP }\end{array}$ & Residual & $\begin{array}{c}\text { Relative } \\
\text { error }(\%)\end{array}$ \\
\hline 1 & 0.268 & 0.268 & 0 & 0 \\
2 & 0.306 & 0.305 & 0 & 0 \\
3 & 0.364 & 0.361 & -0.003 & 0.824 \\
4 & 0.372 & 0.373 & 0.001 & 0.269 \\
5 & 0.423 & 0.420 & -0.003 & 0.71 \\
6 & 0.511 & 0.508 & -0.003 & 0.588 \\
7 & 0.516 & 0.516 & 0 & 0 \\
8 & 0.521 & 0.517 & -0.004 & 0.768 \\
9 & 0.708 & 0.707 & -0.001 & 0.141 \\
10 & 0.768 & 0.767 & -0.001 & 0.13 \\
\hline \multicolumn{3}{c}{$\begin{array}{c}\text { Average relative simulation } \\
\text { percentage error }(\%)\end{array}$} \\
\hline \multicolumn{5}{c}{0.34} \\
\hline
\end{tabular}

From 2016 to 2018, the predicted GDP of the lodging and catering industry in X City is 0.842 and 0.891 and 0.973 , The real value is $0.832,0.901$, and 0.983 .

\subsubsection{The model of Multiple linear regression result}

The results of the multiple linear regression model are shown in Table 3. From Table 3, we can know that the maximum residual error is 0.012 and the Standard error is $0.85 \%$, indicating that the model is reliable. The forecast value for 2016 to 2018 is 0.819 and 0.872 and 0.938 .

Table 3. Results of Multiple linear regression training.

\begin{tabular}{cccc}
\hline number & Actual GDP & $\begin{array}{c}\text { Simulated } \\
\text { GDP }\end{array}$ & Residual \\
\hline 1 & 0.268 & 0.266 & 0.002 \\
2 & 0.305 & 0.304 & 0.001 \\
\hline 3 & 0.364 & 0.362 & 0.002
\end{tabular}

\begin{tabular}{cclc}
4 & 0.372 & 0.374 & -0.002 \\
5 & 0.423 & 0.421 & 0.002 \\
6 & 0.511 & 0.510 & 0.001 \\
7 & 0.516 & 0.520 & -0.004 \\
8 & 0.521 & 0.520 & 0.001 \\
9 & 0.708 & 0.708 & 0 \\
10 & 0.768 & 0.770 & -0.002 \\
\hline Standard error $(\%)$ & \multicolumn{3}{c}{$0.85 \%$} \\
\hline
\end{tabular}

\subsubsection{The model of BP neural network result}

Table 4 shows the results of BP neural network model. It can be seen from Table 4 that the predicted GDP of the lodging and catering industry in X City from 2016 to 2018 is $0.818,0.863$ and 0.876 .

Table 4. Results of BP neural network training.

\begin{tabular}{ccc}
\hline number & Actual GDP & Simulated GDP \\
\hline 1 & 0.268 & 0.269 \\
2 & 0.305 & 0.305 \\
3 & 0.364 & 0.363 \\
4 & 0.372 & 0.375 \\
5 & 0.423 & 0.422 \\
6 & 0.511 & 0.510 \\
7 & 0.516 & 0.515 \\
8 & 0.521 & 0.522 \\
9 & 0.708 & 0.708 \\
10 & 0.768 & 0.767 \\
\hline
\end{tabular}

As shown in Figure 2, the sum of squares of errors tends to be stable when trained to 120 , and as low as 0.0105481 when trained to 140 , which indicates that the results obtained by the model are reliable.

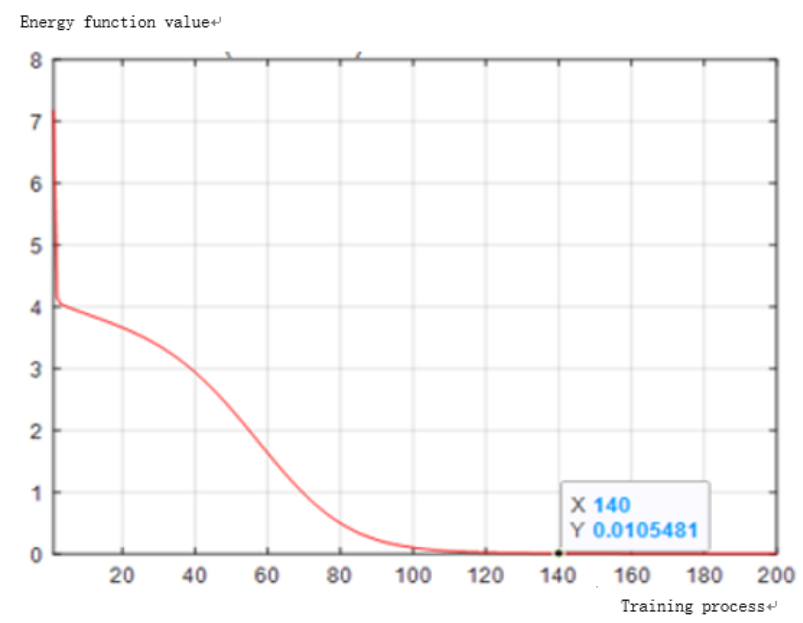

Fig. 2. Change graph of sum of squares of error.

\subsection{Discussion}

Table 5 is the GDP forecast value and related indicators of the lodging and catering industry in X City from 2016 to 2018 , which are obtained by the three forecasting models (GMgdp represents the prediction result of GM $(1, N)$ grey model, BPgdp represents the prediction result 
of BP neural network, and MRgdp represents the prediction result of multiple regression model). From the table, we can know that the forecast result of $\operatorname{GM}(1, \mathrm{~N})$ grey model is closest to the real value, both the value of every time prediction and the average error are smaller than the other two prediction methods. The results of multiple regression model are compared with results of BP neural network model, the accuracy of multiple regression model is better than BP neural network model. The average error of the multiple linear regression model is 2.7 times that of the GM $(1, N)$ grey model, and the average error of the BP neural network model is 4.8 times that of the GM $(1, N)$ grey model, the average error of BP neural network model is 1.9 times that of multiple regression model. In conclusion, $\mathrm{GM}(1, \mathrm{~N})$ grey model has the best performance in the three models, and is suitable for the prediction of future GDP of the lodging and catering industry.

Table 5. Comparison table of prediction results.

\begin{tabular}{|c|c|c|c|c|}
\hline & & GMgdp & MRgdp & BPgdp \\
\hline \multirow{3}{*}{2016} & GDP & 0.842 & 0.819 & 0.817 \\
\hline & $\begin{array}{c}\text { Estimate } \\
\text { value / True } \\
\text { value }\end{array}$ & 1.012 & 0.985 & 0.983 \\
\hline & relative error & 0.012 & 0.015 & 0.017 \\
\hline \multirow{3}{*}{2017} & GDP & 0.891 & 0.872 & 0.863 \\
\hline & $\begin{array}{c}\text { Estimate } \\
\text { value / True } \\
\text { value }\end{array}$ & 0.988 & 0.967 & 0.957 \\
\hline & relative error & 0.012 & 0.033 & 0.043 \\
\hline \multirow{3}{*}{2018} & GDP & 0.973 & 0.938 & 0.876 \\
\hline & $\begin{array}{c}\text { Estimate } \\
\text { value / True } \\
\text { value }\end{array}$ & 0.989 & 0.954 & 0.891 \\
\hline & relative error & 0.011 & 0.046 & 0.109 \\
\hline \multicolumn{2}{|c|}{ average error } & 0.0117 & 0.0313 & 0.0563 \\
\hline
\end{tabular}

\section{Conclusions}

Although the lodging and catering industry is a traditional industry, with the improvement of people's living standards, the situation of the lodging and catering industry is getting better and better, and the role of promoting economic growth is becoming more and more significant. If accurately predict the future output value of lodging and catering industry according to some historical data and external conditions, then the government can implement some policies to promote the healthy development of the industry, and it is conducive to promoting the growth of GDP. However, the GDP of lodging and catering industry in China is generally statistical by quarterly or annual method, and the data volume is relatively small, so it is difficult to accurately predict the future GDP of the industry by common neural network method. In order to study how to accurately predict the GDP of lodging and catering industry in a certain city, this paper uses $\operatorname{GM}(1, N)$ grey model and multiple regression model and BP neural network model to predict the GDP of lodging and catering industry in X city from 2016 to 2018 based on the data of electricity consumption and abnormal weather temperature from 2006 to 2015, find $\operatorname{GM}(1, N)$ grey model has better prediction performance in this industry than regression model and BP artificial network model, and the forecast error is less than $2 \%$.

\section{References}

1. Sheng-Tung Chen, Hsiao-I Kuo, Chi-Chung Chen. The relationship between GDP and electricity consumption in 10 Asian countries[J]. Energy Policy, 2007, 35(4): 2611-2621.

2. A. Ciarreta,A. Zarraga. Economic growth-electricity consumption causality in 12 European countries: A dynamic panel data approach[J]. Energy Policy,2010,38(7).

3. Yoo S H, Kwak S Y. Electricity consumption and economic growth in seven South American countries[J]. Energy Policy, 2010, 38(1): 181-188.

4. Pan Kai. Research on the Relationship between Electricity Consumption and Economic Growth in China [D]. Yunnan University of Finance and Economics,2012.

5. Li Xinying. Econometric analysis of the relationship between electric power and economic growth -Taking Xinjiang as an example [J]. Technological Economics and Management Research,2012(01):9094.

6. Luo Guanglei, Hu Xiguo, Zhao Yang. Forecast analysis of coordinated development between electricity consumption demand and GDP [J]. Contemporary Economy,2014(11):70-72.

7. Sun Yan, Luo X . Empirical consideration on the relationship between GDP and electricity consumption in China $[\mathrm{J}]$. Statistics and Decision,2016(05):98-100.

8. He Yongxiu, Wei Jiajia, Cai Qi, et al. Research on the relationship between electric power and economic growth in Shanxi Province [J]. North China Electric Power Technology, 2007 (4): 1-4.

9. Gao Qian, Liu Yunyun, Ge Yi, Li Bingjie. Forecast of GDP of the tertiary industry in Jiangsu Province based on electric power data [J]. Science, Technology and Economy Guide,2019,27(03):239.

10. Chang Haotian. Research on Macroeconomic Forecasting Method Based on Multi-dimensional Data Fusion of Electricity Consumption [D]. University of Electronic Science and Technology of China,2020.

11. Cao Xuejing, Wang Haixia, Li Tongxing. Application of Multivariable Grey Forecasting Model in Gross National Product Index Forecasting [J]. Journal of Qufu Normal University (Natural Science Edition),2020,46(01):47-51.

12. Shanghai Energy Conservation,2020(03):208-212. DOI: 10.1016 / j.jcip.2016.01.003 SUN Jinpeng. Analysis of Shanghai Power Demand Forecast 
Based on Grey Model [J]. Shanghai Energy Conservation,2020(03):208-212.

13. Wen Xin, Zhang Xingwang, Zhu Yaping, Li Xin. Intelligent Fault Diagnosis Technology: MATLAB Application: Beijing University of Aeronautics and Astronautics Press, 2015.09. 